\title{
Metastatic osteosarcoma in the inferior vena cava and the right atrium
}

W.J. Burger MBChB (Stell)

Registrar at UOFS

\section{Abstract}

\section{Osteosarcoma}

commonly metastasize to the lung, lymph nodes, liver and brain.

In this case we describe a rare presentation of direct infiltration, by an osteosarcoma, of the iliac veins that continued into the inferior vena cava (IVC) and could be seen up to the right atrium.

\section{Introduction}

Osteosarcoma metastases are common and may often present in an uncommon place and fashion. Metastatic cardiac tumours are more common than primary sarcomas of the heart. The incidence is higher in children and young adults and the commonest tumours to metastasize are Wilms' tumours and neuroblastomas. Other abdominal tumours, including secreting and non-secreting adrenal tumours, retroperitoneal sarcomas, hepatocellular carcinomas, teratomas and lymphomas, can involve the IVC.
There have been reports of metastatic osteosarcomas involving the IVC and cardiac chambers.

\section{Case report}

A 31-year-old black woman from Lesotho, southern Africa, presented with a local recurrence of osteosarcoma in her right leg approximately a year-and-a-half after initial diagnosis. The patient had an above knee amputation in December 1998. Oneand-a-half years later she presented with a swollen, firm amputation stump of her right leg. The patient looked chronically ill. The stump showed two small ulcers and a hard mass that could be palpated up to the inguinal area. The patient also had decreased movement of her right hip and on vaginal examination the uterus was immobile. A vague abdominal mass could be felt in the right lower quadrant. No clear lymphadenopathy was found but the patient was slightly anaemic clinically.

\section{Previous medical history}

The patient presented in December 1998 with a swollen mass in her right distal femur that was histologically confirmed to be an osteosarcoma and an above knee amputation was performed. She received chemotherapy. Initially the chest Xray showed no metastases, but she presented three months later with a single pulmonary nodule in her left lung. A metastatectomy was performed. Due to financial constraints, the patient was unable to attend the follow-up Oncology Clinic and presented only six months later with a local recurrence in the area proximal to the amputation of her right leg. A 


\section{Metastatic osteosarcoma in the inferior}

vena cava and the right atrium

\section{from page 10}

chest X-ray also showed new lung metastases in the right lung. (Fig. 1) The tumour was deemed to be unresectable and the patient received three courses of chemotherapy with mixed response.

Again the patient had financial difficulty and only arrived at the Oncology Clinic four months later. Initially, a scintigram was done that was normal. The FBC, SMAC, VDRL, and HIV blood tests were normal.

On the patient's last admission the chest X-ray showed three metastatic nodules in the right lung.

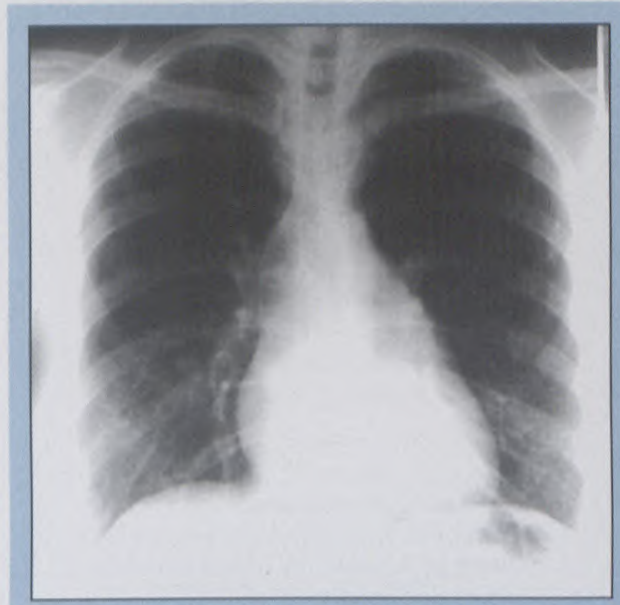

Fig. 1 Chest $X$-ray with a single metastatic nodule in the right lung.

A CT chest, abdomen and pelvis was done before and after intravenous contrast, using a GE spiral scanner with $7 \mathrm{~mm}$ slices and a pitch of 1,7 . Oral gastrografin was also administered one hour before the examination. The CT showed extensive local infiltration of the recurring osteosarcoma in the right upper thigh, inguinal area and right pelvis. It was easily identified by the characteristic calcifications of the osteosarcoma. The bladder, uterus and rectum were displaced to the left. (Fig.2)

Tumour thrombus filled the iliac vein and the IVC and could be traced up to the right atrium. There was clear

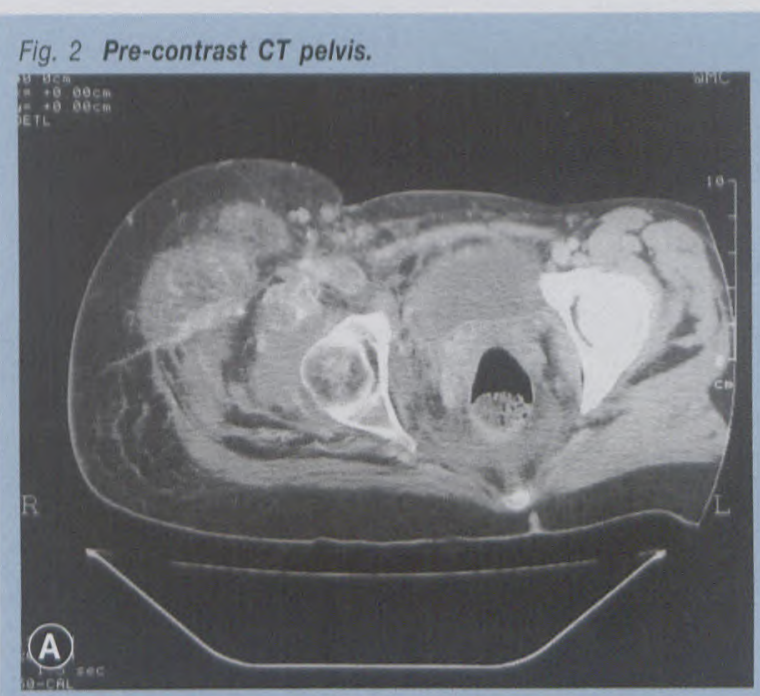

(A) The initial pre-contrast $\mathrm{CT}$ shows a mass with minimal calcifications in the right inguinal area. There are already signs of infiltration of the right femoral vein and collateral veins.

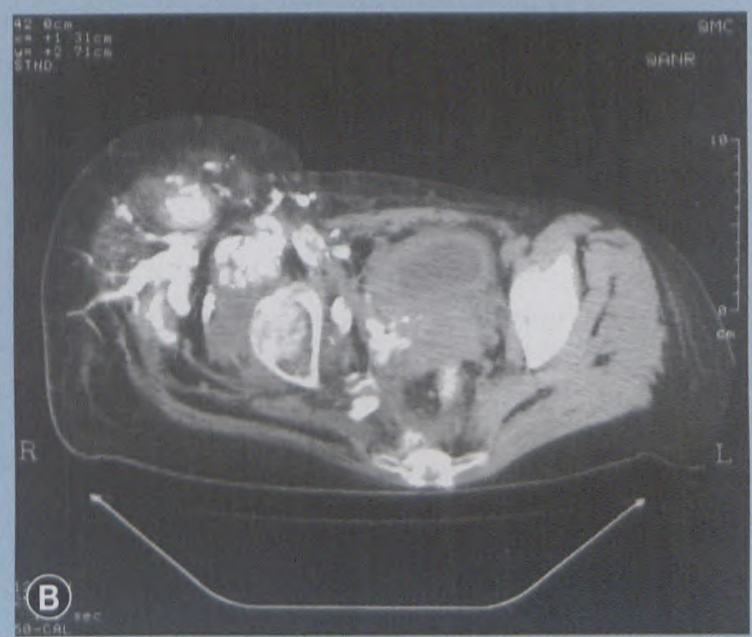

(B) Six months later the $\mathrm{CT}$ was repeated and extensive infiltration into the soft tissue, right femoral vein and collateral veins are seen. The characteristic osteoid calcifications of osteosarcoma are easily identified. veins could be seen throughout the abdomen. There was also hydro-nephrosis and hydro-ureter seen in the right kidney. The right kidney showed a decrease in contrast enhancement and a venous infarct was suspected. (Fig.3)

The spleen and portal vein appeared to be prominent.

The CT chest showed eight small metastatic nodules (Fig. 4), in both the upper and lower lobes of the right lung. There were some postoperative pleuritic changes on the left from the previous metastatectomy.

No clear infiltration of the endocardium of the right atrium could be shown. A prominent azygos vein was seen in the posterior mediastinum. No pleural effusion, liver- or kidneymetastases could be seen.

An ultrasound of the abdomen was done that confirmed the hydro-nephrosis of the right kidney and

expansion of the IVC, especially in the upper abdomen. Infiltration of the IVC wall and extension beyond could not be excluded on CT. On the pre-contrast study the tumour thrombus was filled with calcifications. The tumour thrombus could be seen as a hypodense filling defect, mixed with calcifications in the IVC on the post contrast studies.

The tumour also infiltrated the collateral veins (ascending lumbar veins) that could be seen displacing the right psoas muscle ventrally. Dilated collateral

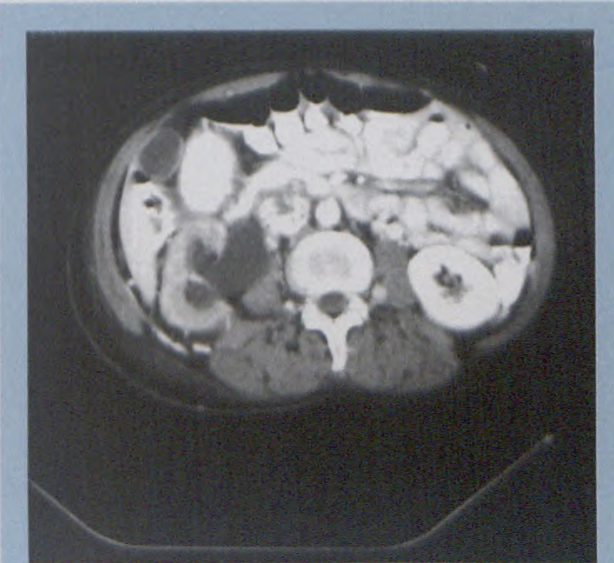

Fig. 3 Post-contrast $\mathrm{CT}$ abdomen. An under enhanced right kidney with a dilated renal pelvis and a degree of hydro-nephrosis could be seen. 
from page 11

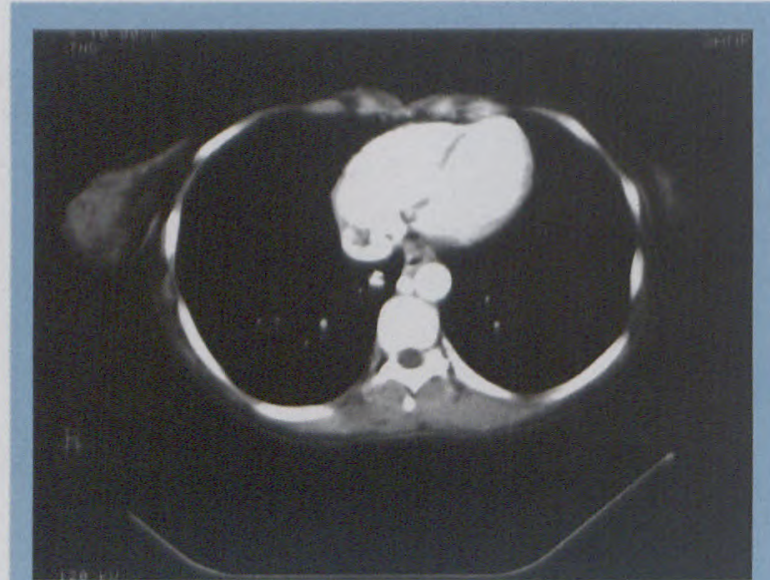

Fig. 4 Post-contrast $\mathrm{Cr}$ chest. A single metastatic nodule is seen on this slice in the right lung. The tumour thrombus extending into the right ventricle could also be seen.
Discontinuous tumour foci may be seen in bone marrow in up to $10 \%$. Other rare sites include the kidneys, ${ }^{2}$ skeletal muscles $^{3}$ and the IVC and heart. ${ }^{4} \mathrm{An}$ isolated brain metastases of osteosarcoma in a patient presenting with a patent foramen ovale has been reported. ${ }^{5}$

Tumour extension to the IVC represents an important complication in abdominal carcinomas, alalso showed a thrombus in the IVC, stretching the whole of the IVC. The thrombus could be visualised in the right atrium.

\section{Discussion}

Osteosarcoma is the most common tumour of bone in adolescents and young adults and the second most common primary bone tumour after multiple myeloma. It accounts for approximately $15 \%$ of all primary bone tumours confirmed at biopsy. There are numerous types of primary osteosarcomas, including intra medullary (high grade, telangiectatic, low grade, small cell, osteosarcomatosis, and gnathic), surface (intracortical, parosteal, periosteal and high grade surface), and extra-skeletal. ${ }^{1}$

Approximately $2 \%$ of patients have metastases at presentation with osteosarcoma. The most common site for metastases is the lung (15\%) via hematogenous spread.

Other sites include lymph nodes, liver and brain. The metastatic lesions may be calcified, although this is rare (10\%). Unlike Ewing's sarcoma, skeletal metastases are uncommon $(<1 \%)$. though very rare in osteosarcomas. The pre-operative diagnosis of vena caval and cardiac involvement is an important consideration in surgical planning and future treatment. The surgical approach and procedure are dependent on the level of superior extension of the tumour thrombus. The following points should be clarified before surgery:

- whether the IVC is involved by tumour thrombus and, if so, the nature of the primary tumour;

- the cranial limit of the tumour thrombus extension, whether it involves the intra-hepatic IVC, the hepatic veins, or the right atrium; and - the presence or absence of tumour invasion of the wall of the IVC. ${ }^{6}$

In patients with musculoskeletal malignancies, thrombosis of the IVC may occur secondary to compression by adjacent adenopathy or by a paraneoplastic hypercoagulable state. Direct extension of the bone tumours into adjacent vessels is, however, rare. The site of tumour entrance into the vessels is not definitely known, although some speculate that with bone tumours the malignant cells enter the veins via intramedullary channels within the bone similar to the entrance route of normal blood-forming elements. ${ }^{4}$

Since the number of patients who benefit from relapse therapy is still low, it remains to be shown whether an increased frequency of lung CTscans or MRIs of the primary tumour site will improve early detection of relapse and, if so, whether that will enhance the chance for successful relapse treatment. Chest X-rays, lung $\mathrm{CT}$ and a clinical examination should be performed routinely for at least three years after completion of therapy or relapse diagnosis. In contrast, bone scintigraphy and local Xrays appear not to be useful as routine follow-up investigations. ${ }^{7}$

\section{References}

1. Atra A, Shankar AG, Padhani AR. Metastatic cardiac osteosarcoma -imaging features. The British Journal of Radiology 1998; 71:336339.

2. Ogose A, Morita T, Emura I, Nemoto K, Hirata Y. Osteosarcoma metastatic to the kidneys without lung involvement [Review]. Japanese Journal of Clinical Oncology 1999; 29(8):395-398.

3. Peh WC, Shek TW, Wang SC, Wong JW, Chien EP. Osteogenic sarcoma with skeletal muscle metastases. Skeletal Radiology 1999; 28(5):298-304.

4. Giuliano CT, Kauffman WM, Haller JO, Fletcher BD, Rao SP. Inferior vena cava-right atrium tumour thrombus in malignant pelvic bone tumours in children. Paediatric Radiology 1992; 22:206-208.

5. Menassa L, Haddad S, Aoun N, Slaba S, Atallah N. Isolated brain metastases in a patient with a patent foramen ovale. European Radiology 1997; 7(3):365-7.

6. Didier D, Racle A, Etienvent JP, Weill F. Tumour thrombus of the inferior vena cava secondary to malignant abdominal neoplasms: US and CT evaluation. Radiology 1987;162:83-89.

7. Korholz D, Verheyen J, Kemperdick HF, Gobel U. Evaluation of follow-up investigations in osteosarcoma patients: Suggestions for an effective follow-up program. Medical and Paediatric Oncology 1998; 30(1): 52-8. 